\title{
Evaluation of the usefulness of a D dimer test in combination with clinical pretest probability score in the prediction and exclusion of Venous Thromboembolism by medical residents
}

\author{
Tarek Owaidah' ${ }^{*}$, Nahlah AlGhasham², Saad AlGhamdi ${ }^{3}$, Dania AlKhafaji ${ }^{3}$, Bandar ALAmro ${ }^{3}$, Mohamed Zeitouni $^{3}$, \\ Fawaz Skaff', Hazzaa AlZahrani ${ }^{5}$, Adher AlSayed ${ }^{5}$, Naser ElKum ${ }^{6}$, Mahmoud Moawad ${ }^{3}$, Ahmed Nasmi ${ }^{3}$, \\ Mohannad Hawari ${ }^{3}$ and Khalid Maghrabi ${ }^{7}$
}

\begin{abstract}
Introduction: Venous thromboembolism (VTE) requires urgent diagnosis and treatment to avoid related complications. Clinical presentations of VTE are nonspecific and require definitive confirmation by imaging techniques. A clinical pretest probability (PTP) score system helps predict VTE and reduces the need for costly imaging studies. D-dimer (DD) assay has been used to screen patients for VTE and has shown to be specific for VTE. The combined use of PTP and DD assay may improve exclusion of VTE and safely avoid imaging studies.

Materials and methods: We prospectively used the Wells PTP score and a DD test to evaluate 230 consecutive patients who presented with VTE symptoms. The receiver operating characteristic curve was used to identify a new DD cutoff value, which was applied to VTE diagnosis and compared with the upper limit of locally established reference range for prediction of thrombosis alone and in combination with the clinical PTP score.

Results: We evaluated 118 patients with VTE symptoms fulfilling the inclusion criteria, 64 (54.2\%) with clinically suspected deep vein thrombosis (DVT) and 54 (45.8\%) with symptoms of pulmonary embolism (PE). The PTP was low in 28 (43.8\%) and moderate/high in 36 (56.25\%) of the suspected DVT patients, and low in 29 (53.7\%) and moderate/high in 25 (46.3\%) of the suspected PE patients. Eighteen cases were confirmed by imaging studies: 9 DVT and 9 PE. The agreement between confirmed cases and PTP was significant with PE but not DVT. The negative predictive value for both DVT and PE with current DD cutoff value of $<250 \mu \mathrm{g} / \mathrm{L}$ DDU was $100 \%$, whereas with the calculated cutoff the NPV was $88 \%$.
\end{abstract}

Conclusions: We confirm that PTP score is valuable tool for medical residents to improve the detection accuracy of VTE, especially for PE. The DD cutoff value of $250 \mu \mathrm{g} / \mathrm{L} \mathrm{FEU} \mathrm{is} \mathrm{ideal} \mathrm{for} \mathrm{excluding} \mathrm{most} \mathrm{cases} \mathrm{of} \mathrm{low} \mathrm{PTP;} \mathrm{however,}$ the calculated cutoff was less specific for the exclusion of VTE.

Keywords: D-dimer, Clinical probability, Deep vein thrombosis, Pulmonary embolism

\footnotetext{
* Correspondence: towaidah@kfshrc.edu.sa

'Department of Pathology and Laboratory Medicine, King Faisal Specialist

Hospital and Research Center, Riyadh, Saudi Arabia

Full list of author information is available at the end of the article
} 


\section{Introduction}

Deep vein thrombosis (DVT) and pulmonary embolism (PE) are common presentations of venous thromboembolism (VTE) that require urgent recognition, diagnosis, and treatment to prevent or minimize the risk of thromboembolic complications and avert the exposure of patients without thrombosis to the risks of anticoagulant therapy and associated morbidity and mortality [1]. Although the clinical symptoms and signs such as dyspnea, pleuritic chest pain, tachypnea, and tachycardia can raise suspicion of PE, and symptoms and signs of swollen, red, tender, and hot lower limbs can raise suspicion of DVT, these are nonspecific and need to be confirmed by further diagnostic and costly imaging techniques [2]. Wells established a clinical prediction rule incorporating signs, symptoms, and risk factors that can accurately be applied to categorize probability for DVT or PE as low, moderate, or high [3]. A recent article suggested that this approach could be further simplified by using only 2 risk categories: DVT unlikely and DVT likely [1]. The Haemostasis and Thrombosis Task Force of the British Committee for Standards in Haematology [4] recommends that to eliminate the need for diagnostic imaging, a combination of D-dimer (DD) assay and pretest probability (PTP) score should give a negative predicative value (NPV) of $>98 \%$, which is equivalent to that of compression ultrasonography for proximal DVT [5].

Unfortunately, a lung scan is often nondiagnostic, even when the incidence of PE ranges from $10 \%$ to $30 \%$, and necessitates further investigations. The PTP score for PE in low, moderate, and high groups has been reported to be $1.3 \%, 16.2 \%$, and $37.5 \%$, respectively [6]. The imaging techniques are costly and operator-dependent, with variability in sensitivity that can be $<73 \%$ in cases of distal DVT [2].

Activation of the coagulation system results in formation of fibrin, which (after being cross-linked by factor XIII) results in a fibrin clot that is subsequently lysed by the activation of the fibrinolytic system, which breaks the fibrin clot to fibrin degradation products. DD is a fibrin-derived fragment that is released into the circulation when cross-linked fibrin is broken down by the fibrinolytic system $[7,8]$. Recently, the DD assay has gained significance as a tool that helps in clinical decisions about the presence of thrombosis. It is generally accepted that clinical assessment and elevated DD levels have further diagnostic advantages, enabling administration of therapy when imaging is not available [9]. On the other hand, several studies have shown that the DD assay may have a high NPV [9-11].

Different techniques are available for DD level measurement, but many lack precision. Enzyme-linked immunosorbent assay (ELISA) continues to be the gold standard for DD level measurement with high sensitivity, but is time-consuming and lacks specificity [8]. New immunological techniques such as immunoturbidimetry have gained attention because of their high sensitivity (around 98\%) with intermediate specificity (better than ELISA) and short process time [12]. The introduction of Point of Care Testing helped further decrease turnaround time; however, poor bioequivalence hampered its use in clinical settings [13]. Both the PTP scoring system and DD assay has resulted in better decision-making and early thrombosis diagnosis $[1,14]$.

In this study, we evaluated the usefulness of PTP scoring system and DD assay as diagnostic tools for frontline clinicians (medical residents) to detect thrombosis in patients presenting with VTE symptoms.

\section{Materials and methods Patients}

Over 3 years, a group of senior medical residents prospectively assessed all inpatients and outpatients $>18$ years of age who presented with suspected PE or DVT for enrollment in this study. Patients were excluded if they had any of the following: 1) a history of venous thrombosis (6 months), 2) recent pelvic surgery (1 month); 3) an indwelling central line; 4) current pregnancy or delivery $<6$ weeks prior; 5 ) inability to undergo compression ultrasonography because of physical or technical limitations; 6) contraindication for radiological contrast; 7) a terminal illness with a life expectancy of $<3$ months; 8) Patients with active cancer; and 9) symptoms that resolved $<72 \mathrm{~h}$ before presentation. The research committee of King Faisal Specialist Hospital and Research Center, Riyadh, Saudi Arabia had approved this study.

\section{Clinical evaluation}

A senior medical resident evaluated all patients clinically at presentation. A data collection form including exclusion criteria, signs, and symptoms included by Wells PTP for DVT and PE was used (Tables 1 and 2) $[1,15]$. Regardless of the PTP score, patients underwent the appropriate imaging technique and a sample was collected for DD testing.

\section{DD testing}

Venous blood was collected by clean venipuncture into $3.2 \%$ sodium citrate to a final ratio of 9:1 using Vacutainer tubes (Becton Dickinson, Franklin Lakes, NJ, USA). Each specimen was centrifuged for $10 \mathrm{~min}$ at $3000 \mathrm{rpm}$ in a refrigerated centrifuge and plasma was drawn into a clean plastic or polystyrene $(12 \times 75 \mathrm{~mm})$ tube and stored at $4^{\circ} \mathrm{C}$ until testing with $24 \mathrm{hr}$. from collection. DD level was tested using Innovance DD (Dade Behring, Marburg, A Siemens Company, Germany)-which is a latex-enhanced, turbidimetric test based on polystyrene 
Table 1 Clinical model for predicting the pretest probability score of deep vein thrombosis (adapted from Wells et al. [1])

\begin{tabular}{|c|c|}
\hline Clinical characteristics & Score \\
\hline $\begin{array}{l}\text { Active cancer (patient receiving treatment for cancer within the } \\
\text { previous } 6 \text { months or currently receiving palliative treatment) }\end{array}$ & 1 \\
\hline $\begin{array}{l}\text { Paralysis, paresis, or recent plaster immobilization of the lower } \\
\text { extremities }\end{array}$ & 1 \\
\hline $\begin{array}{l}\text { Recently bedridden for } \geq 3 \text { days or major surgery within the } \\
\text { previous } 12 \text { weeks requiring general or regional anesthesia }\end{array}$ & 1 \\
\hline $\begin{array}{l}\text { Localized tenderness along the distribution of the deep venous } \\
\text { system }\end{array}$ & 1 \\
\hline Entire leg swollen & 1 \\
\hline $\begin{array}{l}\text { Calf swelling at least } 3 \mathrm{~cm} \text { larger than that on the asymptomatic } \\
\text { side (measured } 10 \mathrm{~cm} \text { below the tibial tuberosity) }\end{array}$ & 1 \\
\hline Pitting edema confined to the symptomatic leg & 1 \\
\hline Collateral superficial veins (nonvaricose) & 1 \\
\hline Previously documented deep vein thrombosis & 1 \\
\hline Alternative diagnosis at least as likely as deep vein thrombosis & -2 \\
\hline
\end{tabular}

particles covalently linked to a monoclonal antibody (DD5) to the cross-linkage region of cross-linked fibrin degradation products. All samples were tested by BCS Instruments from Siemens (Marburg, Germany). The local reference range was established from 20 normal blood bank donors (25-250 $\mu \mathrm{g} / \mathrm{L}$ FEU). All patients had been subjected to radiological studies, Compression ultrasonography for DVT and CT- pulmonary angiography for PE.

Table 2 Rules for predicting the probability of pulmonary embolism (adapted from Wells et al. [15])

\begin{tabular}{lc}
\hline Variable & $\begin{array}{c}\text { No. of } \\
\text { points }\end{array}$ \\
\hline Risk factors & 3.0 \\
Clinical signs and symptoms of deep venous thrombosis & 3.0 \\
An alternative diagnosis deemed less likely than pulmonary & \\
embolism & 1.5 \\
Heart rate >100 beats/min & 1.5 \\
Immobilization or surgery in the previous 4 weeks & 1.5 \\
Previous deep venous thrombosis or pulmonary embolism & 1.0 \\
Hemoptysis & 1.0 \\
Cancer (receiving treatment, treated in the past 6 months, & \\
or palliative care) & \\
Clinical probability & $<2.0$ \\
Low & $2.0-6.0$ \\
Intermediate & $>6.0$ \\
High &
\end{tabular}

\section{Statistics}

Frequency and percentage were used to describe data according to different demographic variables, diagnostic tests, and outcomes for both DVT and PE. Kappa statistic was used to test the agreement between clinical pretest and radiological findings. To obtain the DD cutoff value to rule out DVT and $\mathrm{PE}$, a constructed receiver operating curve (ROC) curve was used considering actual DD results and radiology as the gold standard. The agreement between the new cutoff value and radiology was obtained by the McNemar test. Sensitivity, NPV, specificity, positive predictive value (PPV), and likelihood ratios were calculated on the basis of the diagnostic imaging. $\mathrm{P}<0.05$ was considered significant for all statistical tests. SPSS 20 and Excel 2007 were used for data analysis.

\section{Results}

Of the 230 patients screened during the study enrollment, only 118 fulfilled the inclusion criteria and were included in the analysis. There were 73 (61.9\%) women and $45(38.1 \%)$ men with a mean age at presentation of 52.64 years (range, 17-108 years). The main reasons for exclusion were cancer, an indwelling central line, and pregnancy. The overall prevalence of VTE in this cohort of patients was 15.3\% (18): 9 DVT and 9 PE.

Based on the PTP score, 64 patients (54.2\%) were clinically suspected to have DVT. The most common presentation was a swollen limb in 51 (78.5\%) and lower limb pain in 17 (26.2\%). Fifty-four (45.8\%) were clinically suspected to have PE, with the most common clinical presentations being dyspnea in $42(77.8 \%)$, tachycardia in $14(25.9 \%)$, tachypnea in $14(25.9 \%)$, and pleuritic chest pain in 13 (24.1\%). The PTP score of suspected DVT patients $(\mathrm{n}=64)$ was found to be low in 28 (43.8\%) and moderate to high in 36 (56.2\%), whereas in suspected PE patients $(\mathrm{n}=54)$, it was found to be low in $29(53.7 \%)$ and moderate to high in 25 (46.3\%). The incidence of DVT in patients in low and moderate-to-high PTP score was $7.1 \%$ and $19.4 \%$ respectively, while for PE the incidence was $36 \%$ in moderate-to-high PTP score.

The agreement between PTP score and radiology results for VTE in total (DVT \&PE) was significant $(\mathrm{P}<0.01)$ with sensitivity $88 \%$, specificity $55 \%$, PPV $26 \%$, and NPV $96 \%$. However, sub analysis for DVT and PE showed that it was not significant for suspected DVT $(\mathrm{P}=0.160)$ and significant for suspected PE patients $(P<0.01)$, because all patients with $\mathrm{PE}$ who had been confirmed positive by radiology scored moderate-to-high PTP.

We evaluated the diagnostic value of the established upper limit of the local reference range $(<250 \mu \mathrm{g} / \mathrm{L} \mathrm{FEU})$ alone against radiology results in patients presenting with symptoms suggestive of DVT or PE. There were 96/118 (81.8\%) cases with positive DD but only 18/96 
(19\%) was confirmed by radiology. The calculated sensitivity, specificity, PPV and NPV was 100\%, 22\%, 19\% and $100 \%$ respectively. We assessed the combination of negative DD $(<250 \mu \mathrm{g} / \mathrm{L}$ FEU $)$ with PTP score against radiology, 22/118 (18.6\%) patients were found not to have VTE regardless of the PTP score (Table 3). This is almost the same as using DD result alone.

An ROC curve plotted using the measured DD levels and imaging results was applied to establish a new cutoff value as the point with the highest sum of sensitivity and specificity $(60 \%+63 \%)$ for detecting patients with thrombosis (Figure 1). The area under the curve represents the probability that the assay result for a randomly chosen positive case will exceed the result for a randomly chosen negative case. The asymptotic significance is $<0.05$, which means that results obtained using the assay were better than those obtained without using it.

The calculated DD cutoff value was $815 \mu \mathrm{g} / \mathrm{L}$ FEU for both DVT and PE. To test the accuracy of the calculated cutoff, we used the McNemar test. Considering radiology as the gold standard, we found that the calculated cutoff alone had sensitivity, specificity, PPV and NPV as $88 \%, 58 \%, 19 \%$, and $88 \%$ respectively. The combination of negative calculated DD cutoff $(<815 \mu \mathrm{g} / \mathrm{L}$ FEU) with PTP score identified (56\%) 66/118 cases with negative calculated DD in which (88\%) 58/66 confirmed negative by radiology and (12\%) 8/66 were confirmed positive by radiology (Table 4). These results show that increase in the cutoff of DD showed improvement in PPV with significant reduction in the NPV.

\section{Discussion}

The evaluation of patients with VTE has been improved by the introduction of standardized probability scoring systems. Different PTP scoring systems have been introduced and evaluated for VTE detection sensitivity and specificity and minimizing the need for radiological confirmation [16-18]. Several studies have investigated PTP score assessment in patients with suspected VTE [19-22]. Wells et al developed a score system calculated from clinical and historical data to stratify patients into low,

Table 3 The combination of the negative current DD cutoff and pretest score compared with radiology

\begin{tabular}{llll}
\hline & $\begin{array}{l}\text { Radiology } \\
\text { positive } \\
\text { (Disease } \\
\text { present) }\end{array}$ & $\begin{array}{l}\text { Radiology } \\
\text { negative } \\
\text { (Disease } \\
\text { absent) }\end{array}$ & Total \\
\hline $\begin{array}{l}\text { Test with current cutoff (DD } \\
\text { negative with high PTP score) }\end{array}$ & 0 & $7(30 \%)$ & $7(30 \%)$ \\
$\begin{array}{l}\text { Test with current cutoff (DD } \\
\text { negative with Low PTP score) }\end{array}$ & 0 & $15(70 \%)$ & $15(70 \%)$ \\
Total & 0 & $22(100 \%)$ & $22(100 \%)$ \\
\hline
\end{tabular}

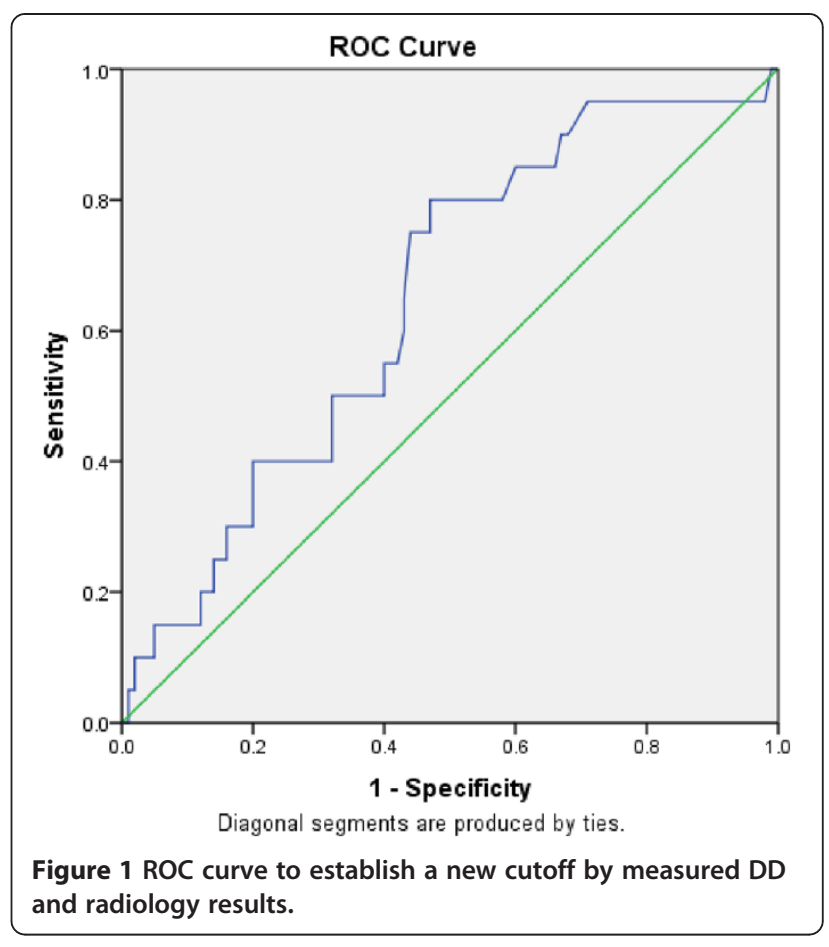

moderate, and high risk of DVT [3]. Other studies have looked at the significance of the value of these PTP systems in predicting PE $[23,24]$. The use of DD, the predominant form of a fibrin-degradation product, as a biomarker of thrombosis had been extensively evaluated clinically in the past several decades [11]. DD sensitivity and specificity were the limiting factors for using this test to evaluate thrombosis. Several studies have shown that ELISA is the most sensitive, but with moderate specificity and high NPV; however, it is been hampered by the impracticality of the time needed to perform $[8,11]$. Many other techniques are available for DD level testing that differ in the principal type of monoclonal antibody specificity that recognizes different epitopes, assay calibration standards, and instrumentation. However, each of these methods has its own limitations

Table 4 Combination between negative calculated DD cutoff and pretest score compared with radiology

\begin{tabular}{llll}
\hline & $\begin{array}{l}\text { Radiology } \\
\text { positive } \\
\text { (Disease } \\
\text { present) }\end{array}$ & $\begin{array}{l}\text { Radiology } \\
\text { negative } \\
\text { (Disease } \\
\text { absent) }\end{array}$ & Total \\
\hline $\begin{array}{l}\text { Test with calculated cutoff } \\
\text { (DD negative with high }\end{array}$ & $8(12.1 \%)$ & $25(37.8 \%)$ & $33(50 \%)$ \\
PTP score) & & $33(50 \%)$ & $33(50 \%)$ \\
$\begin{array}{l}\text { Test with calculated cutoff } \\
\text { (DD negative with low }\end{array}$ & 0 & & \\
PTP score) & & $58(87.8 \%)$ & $66(100 \%)$ \\
Total & $8(12.1 \%)$ & & \\
\hline
\end{tabular}


and predictive value for detecting or excluding thrombosis [25-28].

We used an immunoturbidimetry (Innovance) assay for measuring DD levels in our hospital. It had been reported to have high sensitivity and NPV but low specificity [5]. De Moerloose et al. evaluated Innovance on different analyzers and found it accurate for the VTE diagnosis workup with a sensitivity $>99 \%$ and NPV 95.5\% [29]. In our study, we found that the sensitivity and specificity depends on the selected cutoff value, whereas the reported current DD cutoff value showed $100 \%$ sensitivity and $22 \%$ specificity with a NPV of $100 \%$ and PPV of $19 \%$. These results were also reported in several studies with an NPV range of $91-96 \%$ [25,28,29].

Using a different DD level has been shown to produce a different specificity in excluding VTE. Yamaki et al. showed that the use of ROC curve analysis to select DD cutoff points increased the specificity from $48.9 \%$ to $78.2 \%$ for the low PTP groups; however, it did not achieve substantial improvement in the moderate- and high-risk PTP groups [30]. We did not find improvement in thrombosis detection by using the ROC curve calculated DD level. Courtney et al. showed the same findings when they tested the different DD levels [23]. Although the sensitivity of most new DD assays, including ours, has been shown to be very high, the specificity is low even in ELISA-based assays [2].

The PTP scoring system proposed by Wells was tested in many studies and was found to be reproducible when used by junior residents [31] and could be used to exclude VTE safely when the score was low [3].

Combination of both the PTP score and DD test value has been suggested as sufficiently accurate for the exclusion of VTE and reduction of the requirement for radiology [9-11,14,32,33]. When we combined both PTP score and DD level to exclude VTE, we identified a low PTP score in $43.1 \%$ and moderate to high PTP score in $56.9 \%$ of DVT patients with no significant agreement with radiological studies; however, $52.7 \%$ and $47.3 \%$ of PE patients had a low and moderate-to-high PTP score respectively, with significant agreement of the low PTP score with radiological findings. This can be attributed to more specificity for PE symptoms than for DVT symptoms. Kelly and Hunt found in a pool of data from 4 different studies that $41 \%, 49 \%$, and $10 \%$ of patients had low, moderate, or high PTP score with PE prevalences of $8 \%, 36 \%$, and $67 \%$, which is not very different from our reported results [7].

In conclusion, according to our data, we are certain that we can rule out thrombosis in patients with negative DD results when combined with low PTP score; however, the PTP score is less sensitive for DVT and cannot rule out patients with thrombosis. There is no need to change the cutoff value because the cut off we used based on reference range of DD level showed very high NPV in VTE and the new cutoff value did not considerably improve the PPV alone or in combination with PTP. Our study has a major limitation, which is its low sample size; however, the results are in agreement with those previously reported.

\section{Competing interests}

The authors declare that they have no competing interests.

\section{Authors' contributions}

TO, NG, KM have made substantial contributions to conception and design, SG, DK, BA, MZ, FS, HZ, MM, AN and MH to acquisition of data; TO, NG, KM and $\mathrm{NE}$ to analysis and interpretation of data; TO, NG, KM, SG and BA have been involved in drafting the manuscript or revising it critically for important intellectual content. All authors read and approved the final manuscript.

\section{Acknowledgments}

The authors would like to acknowledge Rashid Nasir for his coordination between the clinical and laboratory team during the time of the study and Areej ALFattani for performing statistical analysis.

\section{Author details}

${ }^{1}$ Department of Pathology and Laboratory Medicine, King Faisal Specialist Hospital and Research Center, Riyadh, Saudi Arabia. ${ }^{2}$ Department of Pathology, College of medicine, Qassim University, Buraidah, Saudi Arabia. ${ }^{3}$ Department of Medicine, King Faisal Specialist Hospital and Research Center, Alfaisal university, Riyadh, Saudi Arabia. ${ }^{4}$ Department of Radiology, King Faisal Specialist Hospital and Research Center, Riyadh, Saudi Arabia. ${ }^{5}$ King Faisal Cancer Centre, King Faisal Specialist Hospital and Research Center, Riyadh, Saudi Arabia. ${ }^{6}$ Department of Biostatistics, Sidra Medical and Research Center, Doha, Qatar. ${ }^{7}$ Department of Critical Care Medicine, King Faisal Specialist Hospital and Research Center, Riyadh, Saudi Arabia.

Received: 22 July 2014 Accepted: 3 November 2014

Published online: 28 November 2014

\section{References}

1. Wells PS, Anderson DR, Rodger M, Forgie M, Kearon C, Dreyer J, Kovacs G, Mitchell M, Lewandowski B, Kovacs MJ: Evaluation of D-dimer in the diagnosis of suspected deep-vein thrombosis. N Engl J Med 2003, 349:1227-1235.

2. Wells PS: Integrated strategies for the diagnosis of venous thromboembolism. J Thromb Haemost 2007, 5(Suppl 1):41-50.

3. Wells PS, Anderson DR, Bormanis J, Guy F, Mitchell M, Gray L, Clement C, Robinson KS, Lewandowski B: Value of assessment of pretest probability of deep-vein thrombosis in clinical management. Lancet 1997, 350:1795-1798.

4. Keeling DM, Mackie IJ, Moody A, Watson HG: Haemostasis and thrombosis task force of the British committee for standards in haematology. The diagnosis of deep vein thrombosis in symptomatic outpatients and the potential for clinical assessment and D-dimer assays to reduce the need for diagnostic imaging. Br J Haematol 2004, 124:15-25.

5. Gardiner C, Pennaneac'h C, Walford C, Machin SJ, Mackie IJ: An evaluation of rapid $\mathrm{D}$-dimer assays for the exclusion of deep vein thrombosis. Br J Haematol 2005, 128:842-848.

6. Wells PS, Anderson DR, Rodger M, Stiell I, Dreyer JF, Barnes D, Forgie M, Kovacs G, Ward J, Kovacs MJ: Excluding pulmonary embolism at the bedside without diagnostic imaging: management of patients with suspected pulmonary embolism presenting to the emergency department by using a simple clinical model and D-dimer. Ann Intern Med 2001, 135:98-107.

7. Kelly J, Rudd A, Lewis RR, Hunt BJ: Plasma D-dimers in the diagnosis of venous thromboembolism. Arch Intern Med 2002, 162:747-756.

8. Stein PD, Hull RD, Patel KC, Olson RE, Ghali WA, Brant R, Biel RK, Bharadia V, Kalra NK: D-dimer for the exclusion of acute venous thrombosis and pulmonary embolism: a systematic review. Ann Intern Med 2004, 140:589-602.

9. Wells PS, Brill-Edwards P, Stevens P, Panju A, Patel A, Douketis J, Massicotte MP, Hirsh J, Weitz JI, Kearon C: A novel and rapid whole-blood assay for 
D-dimer in patients with clinically suspected deep vein thrombosis. Circulation 1995, 91:2184-2187.

10. Freyburger G, Trillaud H, Labrouche S, Gauthier P, Javorschi S, Bernard P, Grenier N: D-dimer strategy in thrombosis exclusion-a gold standard study in 100 patients suspected of deep venous thrombosis or pulmonary embolism: 8 DD methods compared. Thromb Haemost 1998, 79:32-37.

11. Brill-Edwards P, Lee A: D-dimer testing in the diagnosis of acute venous thromboembolism. Thromb Haemost 1999, 82:688-694.

12. Adam SS, Key NS, Greenberg CS: D-dimer antigen: current concepts and future prospects. Blood 2009, 113:2878-2887. doi:10.1182/blood2008-06-165845. Epub 2008 Nov 13. Review.

13. Perveen $S$, Unwin D, Shetty AL: Point of care D-dimer testing in the emergency department: a bioequivalence study. Ann Lab Med 2013, 33:34-38. doi:10.3343/alm.2013.33.1.34. Epub 2012 Dec 17.

14. Peter FF, Victor FT: The evaluation of suspected pulmonary embolism. N Engl J Med 2003, 349:1247-1256.

15. Wells PS, Anderson DR, Rodger M, Ginsberg JS, Kearon C, Gent M, Turpie AG, Bormanis J, Weitz J, Chamberlain M, Bowie D, Barnes D, Hirsh J: Derivation of a simple clinical model to categorize patients, probability of pulmonary embolism: increasing the models, utility with the SimpliRED D-dimer. Thromb Haemost 2000, 83:416-420.

16. Kelly L, Hunt BJ: The utility of pretest probability in patient with clinically suspected thromboembolism. J Thromb Haemost 2003, 1:1888-1896.

17. van der Velde EF, Toll DB, Ten Cate-Hoek AJ, Oudega R, Stoffers HE, Bossuyt PM, Büller HR, Prins MH, Hoes AW, Moons KG, van Weert HC: Comparing the diagnostic performance of 2 clinical decision rules to rule out deep vein thrombosis in primary care patients. Ann Fam Med 2011, 9:31-36.

18. van Es J, Mos I, Douma R, Erkens P, Durian M, Nizet T, van Houten A, Hofstee $H$, ten Cate H, Ullmann E, Büller H, Huisman M, Kamphuisen PW: The combination of four different clinical decision rules and an ageadjusted D-dimer cut-off increase the number of patients in whom acute pulmonary embolism can safely be excluded. Thromb Haemost 2012, 107:167-171.

19. Musset $D$, Parent F, Meyer G, Maitre S, Girard P, Leroyer C, Revel MP, Carette MF, Laurent M, Charbonnier B, Laurent F, Mal H, Nonent M, Lancar R, Grenier P, Simonneau G: Diagnostic strategy for patients with suspected pulmonary embolism: a prospective multicenter outcome study. Lancet 2002, 360(9349):1914-1920.

20. Perrier A, Miron MJ, Desmarais $S$, de Moerloose P, Slosman D, Didier D, Unger PF, Junod A, Patenaude JV, Bounameaux H: Using clinical evaluation and lung scan to rule out suspected pulmonary embolism: is it a valid option in patients with normal results of lower-limb venous compression ultrasonography? Arch Intern Med 2000, 160:512-516.

21. Sanson BJ, Lijmer JG, Mac Gillavry MR, Turkstra F, Prins MH, Büller HR: Comparison of a clinical probability estimate and two clinical models in patients with suspected pulmonary embolism. ANTELOPE-Study Group. Thromb Haemost 2000, 83(2):199-203.

22. Kelly J, Hunt BJ: The utility of pretest probability assessment in patients with clinically suspected venous thromboembolism. J Thromb Haemost 2003, 1:1888-1896

23. Kline JA, Courtney DM, Than MP, Hogg K, Miller CD, Johnson CL, Smithline HA: Accuracy of very low pretest probability estimates for pulmonary embolism using the method of attribute matching compared with the Wells score. Acad Emerg Med 2010, 18(2):133-141.

24. Biss TT, Brandão LR, Kahr WH, Chan AK, Williams S: Clinical probability score and D-dimer estimation lack utility in the diagnosis of childhood pulmonary embolism. J Thromb Haemost 2009, 7:1633-1638.

25. Luxembourg B, Schwonberg J, Hecking C, Schindewolf M, Zgouras D, Lehmeyer S, Lindhoff-Last E: Performance of five D-dimer assays for the exclusion of symptomatic distal leg vein thrombosis. Thromb Haemost 2012, 107:369-378. doi:10.1160/TH11-07-0511. Epub 2012 Jan 11.

26. Prisco D, Grifoni E: The role of D-dimer testing in patients with suspected venous thromboembolism. Semin Thromb Hemost 2009, 35(1):50-59.

27. Dempfle CE, Zips S, Ergül H, Heene DL: Fibrin assay comparative trial study group. The Fibrin Assay Comparison Trial (FACT): evaluation of 23 quantitative D-dimer assays as basis for the development of D-dimer calibrators. FACT study group. Thromb Haemost 2001, 85:671-678.

28. Van der Graaf F, van den Borne $H$, van der Kolk M, de Wild PJ, Janssen GW van Uum SH: Exclusion of deep venous thrombosis with D-dimer testing Comparison of 13 D-dimer methods in 99 outpatients suspected of deep venous thrombosis using venography as reference standard. Thromb Haemost 2000, 83:191-198.

29. De Moerloose P, Palareti G, Aguilar C, Legnani C, Reber G, Peetz D: A multicenter evaluation of a new quantitative highly sensitive D-dimer assay for exclusion of venous thromboembolism. Thromb Haemost 2008, 100:505-512.

30. Yamaki T, Nozaki M, Sakurai H, Kikuchi Y, Soejima K, Kono T, Hamahata A, Kim K: Combined use of pretest clinical probability score and latex agglutination D-dimer testing for excluding acute deep vein thrombosis. J Vasc Surg 2009, 50:1099-1105.

31. Penaloza A, Laureys M, Wautrecht JC, Lheureux P, Motte S: Accuracy and safety of pretest probability assessment of deep vein thrombosis by physicians in training using the explicit Wells clinical model. J Thromb Haemost 2006, 4:278-281.

32. Kearon C, Ginsberg JS, Douketis J, Crowther M, Brill-Edwards P, Weitz J, Hirsh J: Management of suspected deep venous thrombosis in outpatients by using clinical assessment and D-dimer testing. Ann Intern Med 2001, 135:108-111.

33. Janes S, Ashford N: Use of a simplified clinical scoring system and D-dimer testing can reduce the requirement for radiology in the exclusion of deep vein thrombosis by over $20 \%$. Br J Haematol 2001, 112:1079-1082.

doi:10.1186/s12959-014-0028-7

Cite this article as: Owaidah et al:: Evaluation of the usefulness of a D dimer test in combination with clinical pretest probability score in the prediction and exclusion of Venous Thromboembolism by medical residents. Thrombosis Journal 2014 12:28.

\section{Submit your next manuscript to BioMed Central and take full advantage of:}

- Convenient online submission

- Thorough peer review

- No space constraints or color figure charges

- Immediate publication on acceptance

- Inclusion in PubMed, CAS, Scopus and Google Scholar

- Research which is freely available for redistribution 